\title{
MicroRNA-27a regulates the viability, migration and invasion of human skin cancer cells by targeting MAPK7
}

Lei Cao ${ }^{1}$, Ping Luo ${ }^{2}$, Hui Wang ${ }^{3}$, Feiyan Rao ${ }^{1}$

\author{
${ }^{1}$ Department of Dermatology, The First People's Hospital of Fuyang Hangzhou, \\ Hangzhou, China \\ 2Department of Gynaecology, The First People's Hospital of Fuyang Hangzhou, \\ Hangzhou, China \\ ${ }^{3}$ Department of Pathology, The First People's Hospital of Fuyang Hangzhou, \\ Hangzhou, China
}

Submitted: 9 February 2020

Accepted: 28 March 2020

Arch Med Sci

DOI: https://doi.org/10.5114/aoms.2020.95962

Copyright @ 2020 Termedia \& Banach

\section{Abstract}

Introduction: This study was undertaken to determine the expression profile of microRNA-27a (miR-27a) in human skin cancer and to assess its effects on cell viability, migration and invasion.

Material and methods: Expression analysis was performed by GRT-PCR. Transfections were carried out using Lipofectamine 2000 reagent. AO/EB, DAPI, and Annexin V/PI staining was used for detection of apoptosis. Western blot analysis was performed to detect protein expression.

Results: The results showed up to 7 -fold upregulation of miR-27a in skin cancer tissues and up to 5 -fold upregulation in skin cancer cell lines. Inhibition of miR-27a resulted in depletion of A431 cell viability as revealed by MTT assay. Phase-contrast microscopy and AO/EB assay showed signs of apoptosis in A431 cells. Immunohistochemical analysis showed reduction of the $\mathrm{Bcl}-2$, p53 and ki-67 expression in A431 cells. Western blot analysis showed an increase in Bax and caspase- 3 and a decrease in $\mathrm{Bcl}-2$ protein expression. The wound healing and transwell assays revealed a remarkable decline of cell migration and invasion upon miR-27a inhibition. The bioinformatics and luciferase assays showed that miR-27a targets MAPK7. The Western blot analysis showed that MAPK7 was significantly downregulated in all skin cancer cells. Nonetheless, the expression of MAPK7 was significantly decreased upon miR-27a inhibition. Furthermore, MAPK7 overexpression increased the viability of A431 cells. However, MAPK1 silencing could avoid the suppressive effects of miR-27a inhibition on A431 cells.

Conclusions: The evidence suggests that miR-27a may find use in the treatment of skin cancer and warrants further investigation.

Key words: microRNA, apoptosis, viability, MAPK7, skin cancer, migration.

\section{Introduction}

A major health concern worldwide, skin cancer is one of the destructive types of cancers responsible for a huge number of deaths [1]. As per reports, out of every 5 Americans, one develops skin cancer any time during their life. Surgery and chemotherapy are generally employed for skin cancer treatment. In certain cases, the combination of chemo- and radiotherapy is also used for its management [2]. Despite recent advances, the incidence of skin cancer is increasing at an alarming rate. Additionally, late

\author{
Corresponding author: \\ Lei Cao \\ Department \\ of Dermatology \\ The First People's Hospital \\ of Fuyang Hangzhou \\ Hangzhou 311400 \\ China \\ Phone/fax: \\ +86-0571-63157569 \\ E-mail: fycaolei@163.com
}


diagnosis and frequent relapses add to the problems faced during skin cancer treatment. The lack of molecular therapeutic targets and biomarkers for early diagnosis is a further hurdle to proper skin cancer treatment [3]. Over the last few decades, microRNAs (miRs) have gained importance in cancer research and are believed to play essential roles in cancer treatment [4]. The miRs regulate diverse processes in human cells, such as differentiation, proliferation and programmed cell death to name a few [5]. A vast number of studies have confirmed that miR expression levels are remarkably dysregulated in cancer cells. These miRs either promote (oncomiRs) or suppress (tumour suppressor) the progression and growth of cancers [6]. Many miRs have also been shown to act as molecular markers for detection and cancer progression [7]. miR-27a has been shown to be involved in the regulation of growth and metastasis of different cancer types. miR-27a has been shown to act as an oncogene in adenocarcinoma [8]. In pancreatic cancer, miR-27a targets Sprouty2 to control its proliferation [9]. In osteosarcoma, miR-27a modulates the expression of MAP2K4 to suppress its growth [10]. Uveal melanoma growth is inhibited by genistein via inhibition of miR-27a [11]. Despite all these studies, the role of miR-27a has been studied in skin cancer. Against this backdrop, the present study was undertaken to study the function and prospective therapeutic implications of miR-27a in skin cancer. The results showed that miR-27a acts as an oncomiR in skin cancer and may have therapeutic implications in skin cancer treatment.

\section{Material and methods}

\section{Cell lines, tissues and culture conditions}

Skin cancer tissue specimens and normal adjacent tissues of fifteen skin cancer patients who underwent surgical resection in the First People's Hospital of Fuyang Hangzhou, Hangzhou, China from May 2017 to June, 2019 were included. Patients were aged 30-69 years with an average age of 44.20 years. The skin cancer cell lines (A431, A-375, A-101D, HMCB, Hf688(A).T) and the normal skin cell line (HEMn-LP) were procured from Type Culture Collection of Chinese Academy of Sciences, Shanghai, China. The cells were cultured in RPMI-1640 medium containing $10 \%$ fetal bovine serum, $100 \mu \mathrm{g} / \mathrm{ml}$ streptomycin and $100 \mathrm{U} / \mathrm{ml}$ penicillin and a humidified atmosphere containing $5 \% \mathrm{CO}_{2}$. The study was approved by the research ethics committee of the institute under the approval number 66578-FFH of 2019.

\section{qRT-PCR}

The RNA was extracted from the transfected A431 cells using Trizol regent and subsequently
cDNA was synthesized using a RevertAid cDNA synthesis kit. The cDNA was then used as a template for RT-qPCR analysis with the assistance of the Taq PCR Master Mix kit (Qiagen, Inc.) according to the manufacturer's protocol. For determination of the expression, the cycling conditions were as follows: $95^{\circ} \mathrm{C}$ for $15 \mathrm{~s}$, followed by 40 cycles of $95^{\circ} \mathrm{C}$ for $20 \mathrm{~s}$, and $55^{\circ} \mathrm{C}$ for $1 \mathrm{~min}$. GAPDH was used as an internal control. The relative expression was determined by $\Delta \Delta C T$ methodology as described previously [12].

\section{Transfection}

The transfection of miR-NC, NC, miR-27a Inh (inhibitor), pcDNA-MAPK7 and si-MAPK7 were performed with Lipofectamine 2000 (Invitrogen) following user guidelines.

\section{Cell viability assay}

The transfected A431 cells $\left(2.5 \times 10^{3}\right)$ were cultured in each 96-well plate. Following incubation of 3 days, the cells were subjected to treatment with $25 \mu$ of MTT. The medium was removed after about $4 \mathrm{~h}$ and DMSO $(170 \mu \mathrm{l})$ was added to the culture to enable the solubilisation of the formazan crystals. Finally, the absorbance was taken at $570 \mathrm{~nm}$ for the determination of A431 cell viability.

\section{Phase contrast microscopy}

The transfected A431 cells were evaluated for changes in morphology by Olympus BX41 (magnification; 400x) and digital images of the cells were taken.

\section{Acridine orange/ethidium bromide (AO/EB) staining}

The transfected A431 cells were used for the assessment of the nuclear morphology after staining with a solution of $A O / E B$ for $25 \mathrm{~min}$ at $20^{\circ} \mathrm{C}$. After fixation with methanol (70\%), the nuclear morphology was investigated by fluorescence microscopy. Five different random fields were selected for fluorescence microscopy. The annexin V-FITC assay was performed as determined previously [13].

\section{Immunocytochemical analysis}

Firstly the transfected A431 cells were cultured on coverslips until $70 \%$ confluence was achieved. The avidin-biotin complex immunoperoxidase methodology was employed for immunohistochemical analysis of the cells. The Bcl-2, Ki-67, caspase- 3 and p53 expression was assessed using the respective monoclonal antibodies. Ten random fields were selected for analysis 
using an Olympus BX 41microscope with 400x magnification.

\section{Migration and invasion assay}

Transwell chamber with Matrigel coating was used to assess the invasion of transfected cancer cells. Briefly, a $100 \mu$ cell culture containing 6000 cells was added to the upper chamber of the transwell and lower chamber was given $750 \mu \mathrm{l}$ of DMEM medium supplemented with $10 \%$ FBS. After $48 \mathrm{~h}$ incubation at $37^{\circ} \mathrm{C} / 5 \% \mathrm{CO}_{2}$, cells from the surface of the membrane's upper side were removed carefully with cotton swabs while those that adhered to the lower side of the membrane were fixed with $70 \%$ ethyl alcohol and stained with $0.1 \%$ crystal violet. A light microscope (100x) was used for visualization of cells and photographs were taken. At least seven random fields were used for counting of invasive cells. The wound heal assay was used to determine the cell migration as described earlier [13].

\section{Statistical analysis}

The experiments were performed in triplicate and expressed as mean \pm SD. One-way ANOVA

A

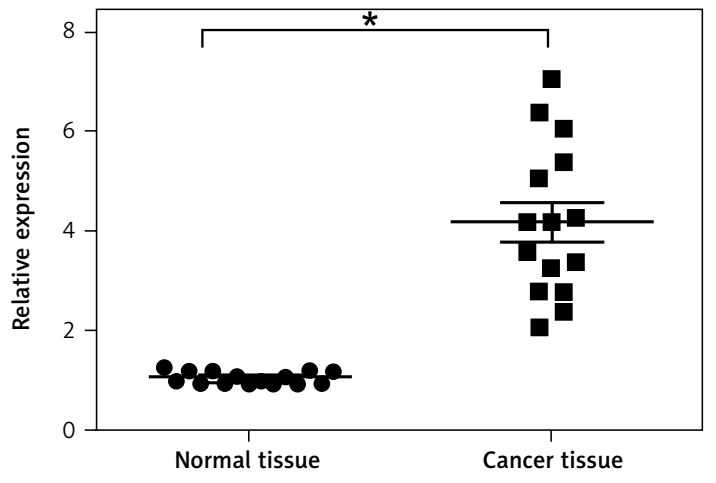

C

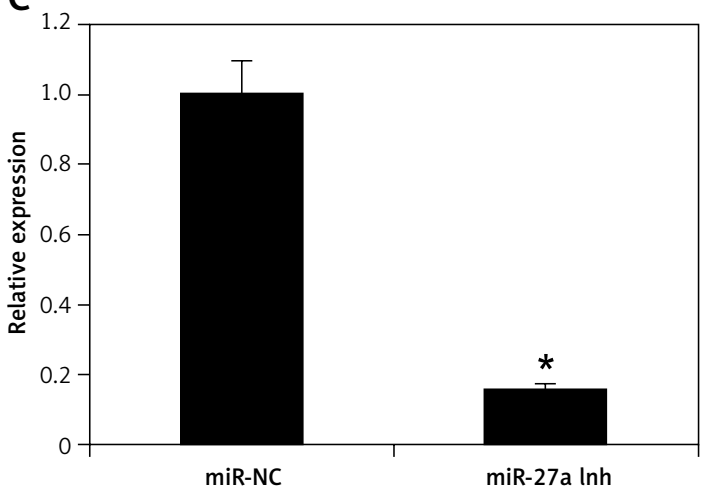

followed by Tukey's $t$ test was used for statistical analysis using GraphPad prism 7 software. P-values $<0.05$ were considered to indicate a statistically significant difference.

\section{Results}

\section{Upregulation of miR-27a in skin cancer}

The experiments started with the assessment of the gene expression of miR-27a in the skin cancer tissues and the adjacent normal tissues by qRT-PCR. The outcomes of the qRT-PCR as determined by $\triangle \triangle C T$ methodology revealed remarkable upregulation of miR-27a in skin cancer tissues relative to adjacent normal tissues (Figure $1 \mathrm{~A}$ ). The upregulation was up to more than 7-fold. Subsequently, the transcripts of miR-27a were also examined in skin cancer cell lines (A431, A-375, A-101D, HMCB, Hf688(A).T) and the normal skin cell line (HEMn-LP) and, interestingly, the results were similar. The expression of miR-27a was significantly enhanced in the skin cancer cells, with the highest upregulation of up to 5.1-fold in A431 cells (Figure $1 \mathrm{~B}$ ). This aberrant expression of miR27 a encouraged us to further study the function of miR-27a in skin cancer.

\section{B}

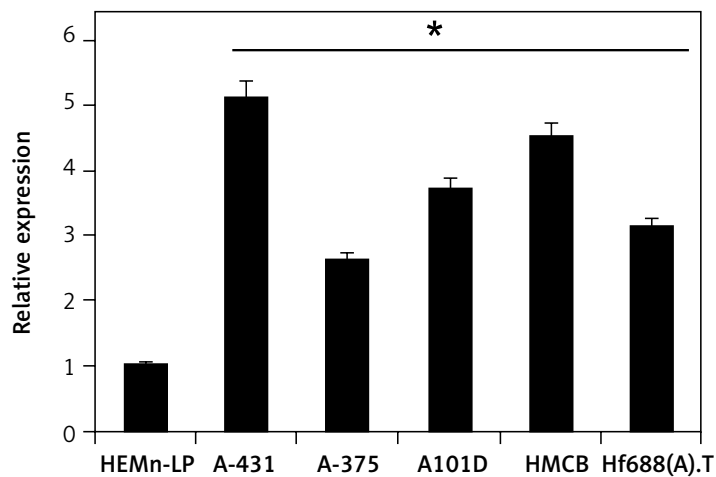

D

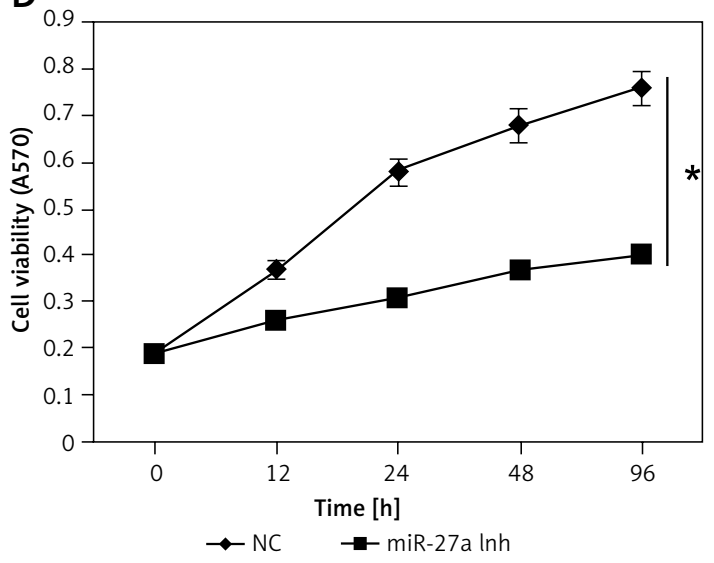

Figure 1. Expression of miR-27a in (A) skin cancer and normal adjacent tissues (B) normal and skin cancer cell lines. C - Expression of miR-27a in miR-NC and miR-27a inhibitor transfected A431 cells. D - Viability of miR-NC and miR-27a inhibitor transfected A431 cells. The experiments were performed in triplicate and expressed as mean \pm SD $\left({ }^{*} p<0.05\right)$ 
miR-27a regulates apoptosis of skin cancer cells

Since miR-27a was remarkably upregulated in both skin cancer tissues and cell lines, we inhib-

A
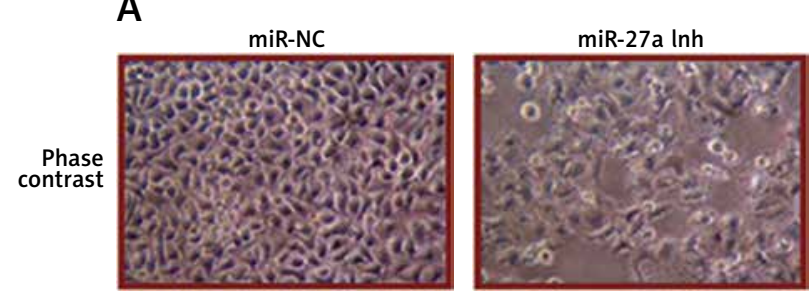

AO/EB
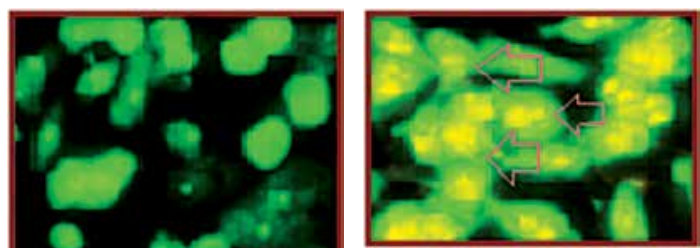

$\mathrm{Bcl}-2$
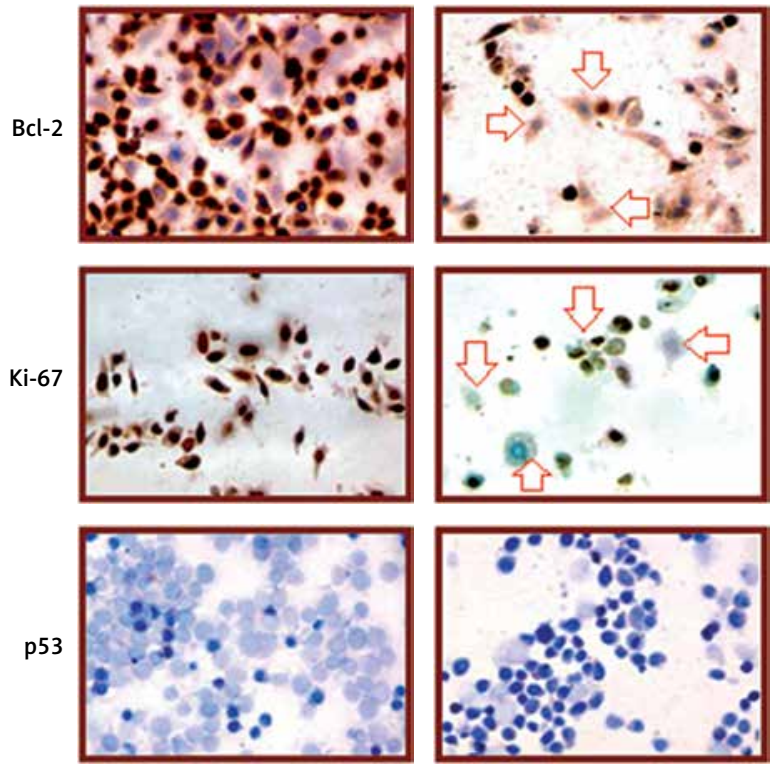

C
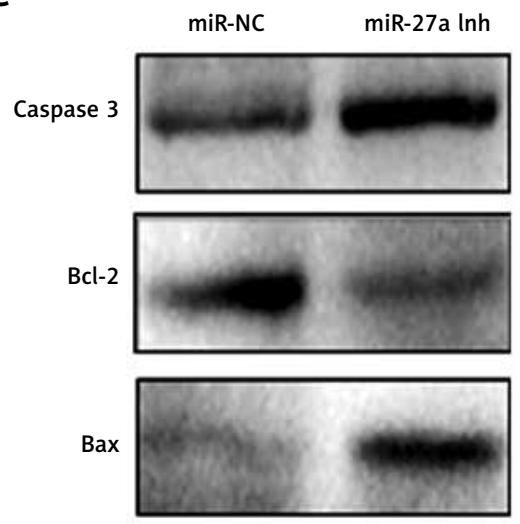

GAPDH

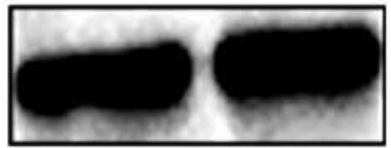

ited the expression of miR-27a to reveal its effects on A431cell viability. Interestingly, the MTT assay showed a time-dependent depletion of the A431 cell viability as the expression of miR-27a was inhibited (Figures 1 C, D). Secondly, different

B
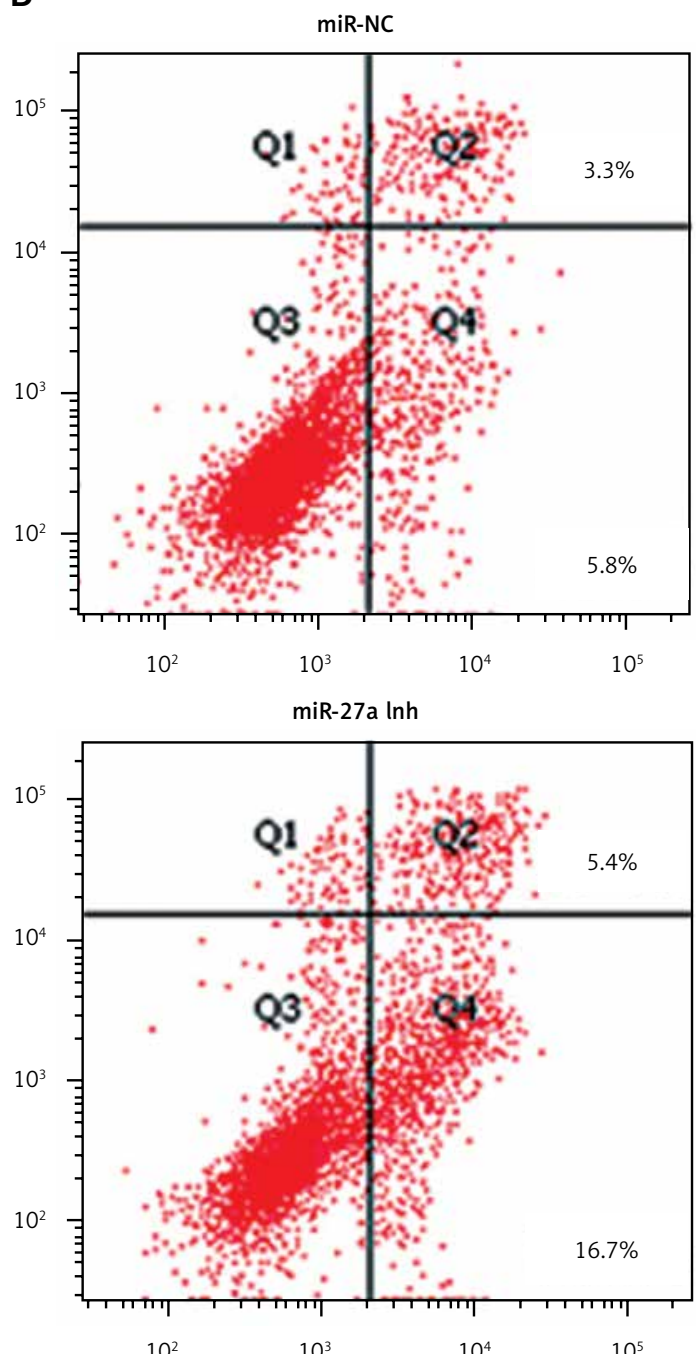

$10^{2}$

$10^{3}$

$10^{5}$

Figure 2. A - miR-NC and miR-27a inhibitor transfected A431 cells showing morphology as revealed by phase contrast microscopy, induction of apoptosis as depicted by AO/EB staining and immunohistochemical analysis showing expression of $\mathrm{Bcl}-2$, $\mathrm{Ki}-67$ and p53. Annexin V/PI staining of miR-NC and miR-27a inh transfected A431 cells (B) and miR-NC and miR-27a inh transfected A431 cells showing the expression of caspase-3, Bcl-2 and Bax (C). The experiments were performed in triplicate 
assays such as phase contrast microscopy and AO/EB staining showed signs of apoptosis in the transfected A431 cells (Figure $2 \mathrm{~A}$ ). The immunohistochemical analysis showed a considerable decrease in the expression of $\mathrm{Bcl}-2, \mathrm{ki}-67$ and p-53 proteins, suggesting the induction of apoptosis in A431 cells (Figure 2 A). The annexin V/ $\mathrm{PI}$ assay showed around $17 \%$ apoptosis in miR27 a Inh transfected cells relative to $6 \%$ in $\mathrm{miR}$ NC transfected cells (Figure $2 \mathrm{~B}$ ). The western blot analysis also revealed a decrease in $\mathrm{Bcl}-2$ and increase in caspase- 3 and Bax expression upon miR-27a inhibition in A431 cells (Figure 2 C). All these outcomes clearly indicate that suppression of miR-27a in A431 cells promotes apoptosis in A431 cells.

\section{miR-27a regulates the migration and invasion of skin cancer cells}

Next, to analyse whether miR-27a has any role in metastasis of A431 cells, we performed the wound healing and transwell assays. The wound healing assay showed remarkable and significant inhibition of the cell migration as obvious from the wound width (Figure $3 \mathrm{~A}$ ). The transwell chamber assay revealed around $73 \%$ inhibition of $A 431$ cell invasion upon miR-27a inhibition (Figure 3 B), indicating the regulation of A31 cell migration and invasion by miR-27a.

\section{miR-27a exerts its effects via MAPK7 signalling}

The informatics revealed miR-27a targets MAPK7 (Figure 4 A). The dual luciferase showed a strong interaction between miR-27a and MAPK7 (Figure $4 \mathrm{~B}$ ). The western blot analysis showed that MAPK7 was remarkably suppressed in all the skin cancer cell lines (Figure $4 \mathrm{C}$ ). Nonetheless, the expression of MAPK7 was considerably increased upon suppression of miR-27a (Figure 4 D). Similar results were also obtained by qRT-PCR (Figures $4 \mathrm{E}, \mathrm{F})$. Furthermore, MAPK7 overexpression also reduced the A431 cell viability time dependently (Figure $4 \mathrm{G}$ ). Nonetheless, silencing of MAPK7 could avoid the growth inhibitory effects of miR$27 \mathrm{a}$ inhibition (Figure $4 \mathrm{H}$ ). All these findings point towards the role of MAPK7 signalling in miR-27a mediated effects on A431 cells.

\section{Discussion}

Skin cancers constitute one of the prevalent types of human cancer and therefore are responsible for tremendous human mortality across the world [14]. The rising incidence rates of skin can-
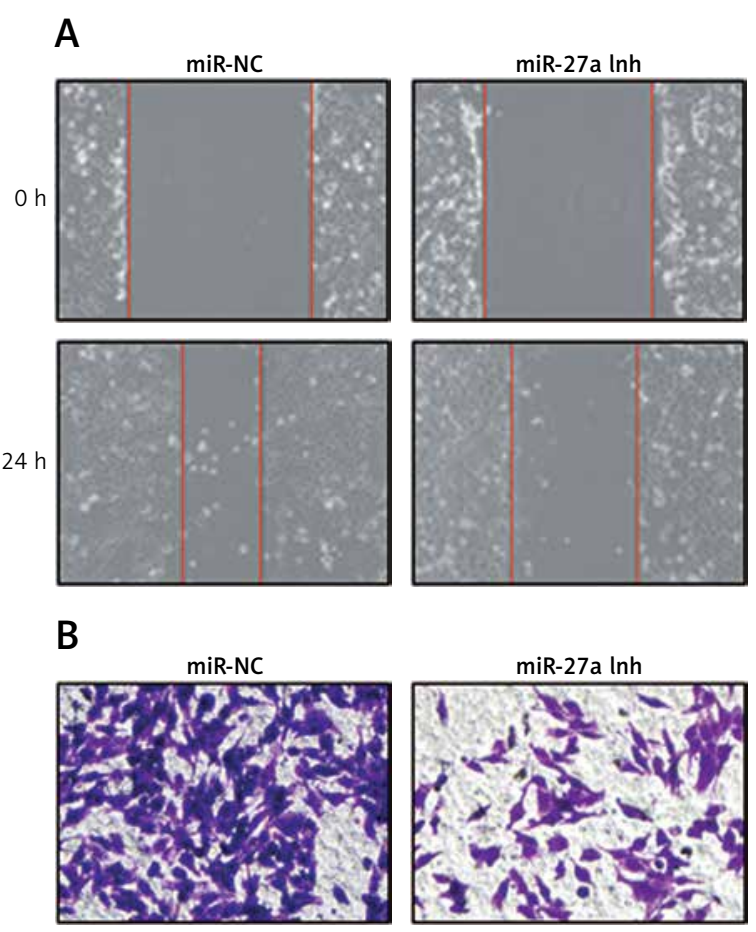
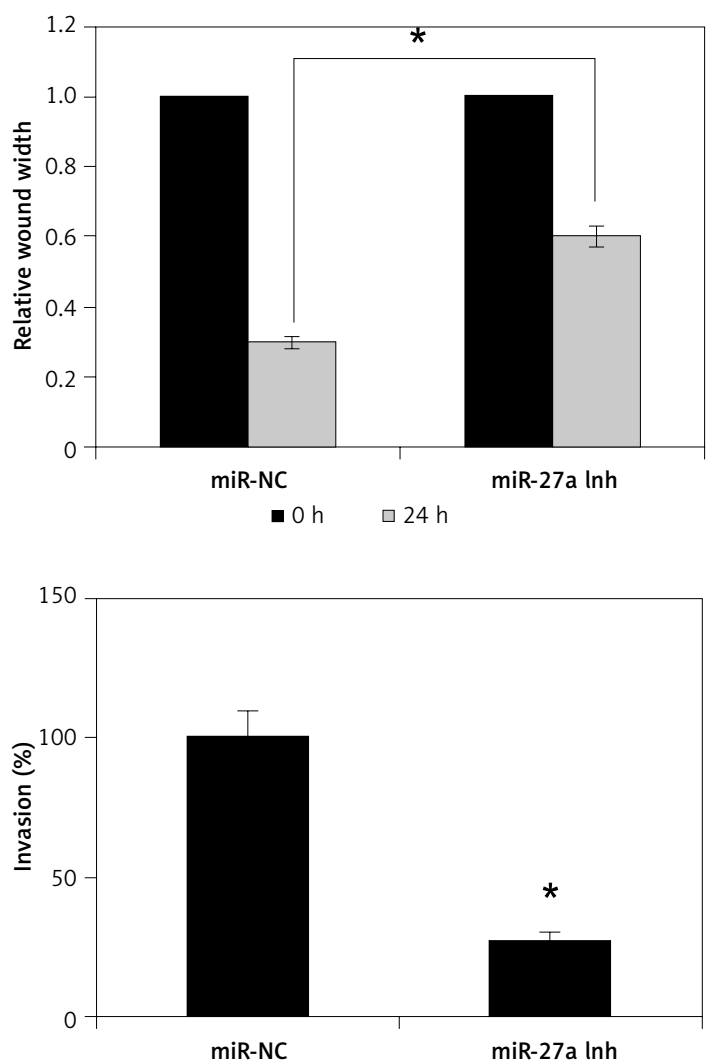

Figure 3. A - Wound healing assay showing cell migration of the miR-NC and miR-27a inhibitor transfected A431 cells. B - Transwell assay showing cell invasion of miR-NC and miR-27a inhibitor transfected A431 cells. The experiments were performed in triplicate and expressed as mean \pm SD $\left({ }^{*} p<0.05\right)$ 
A

miR-27a 3'-GCCU-UGAAUCGGU GACACUU -5'

C

HEMn-LP A-431 A-375 A101D HMCB Hf688(A).T

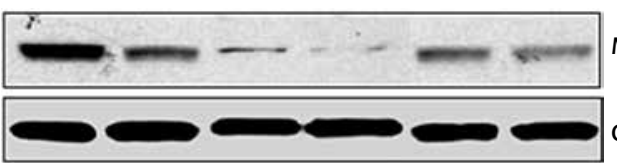

MAPK7

D

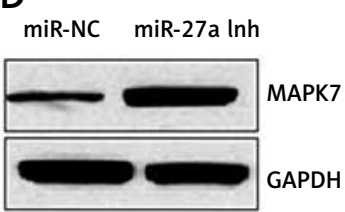

F

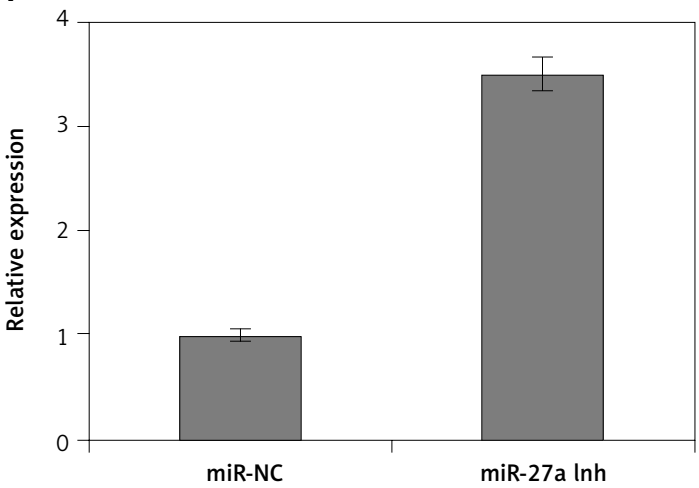

$\mathrm{H}$

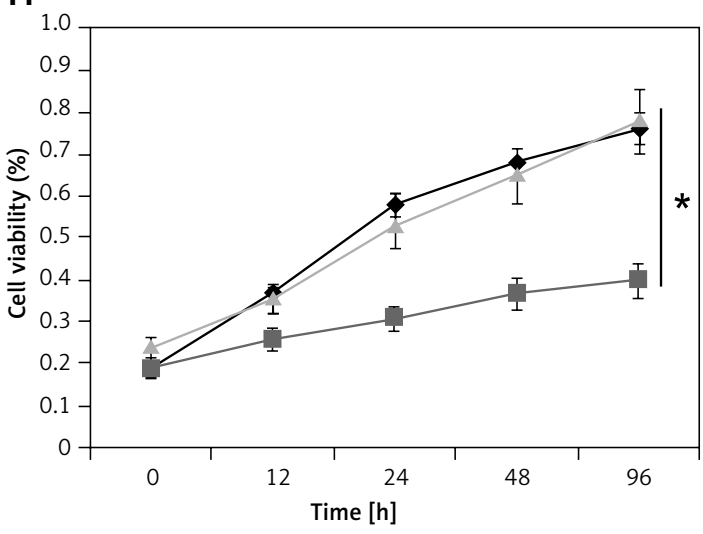

B

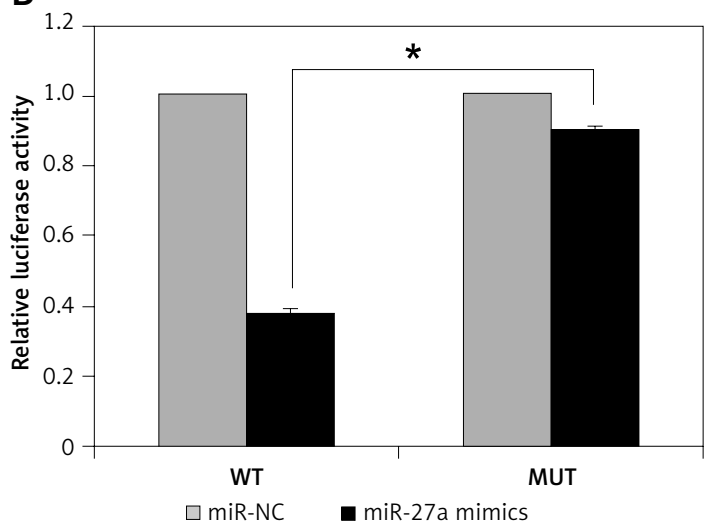

E

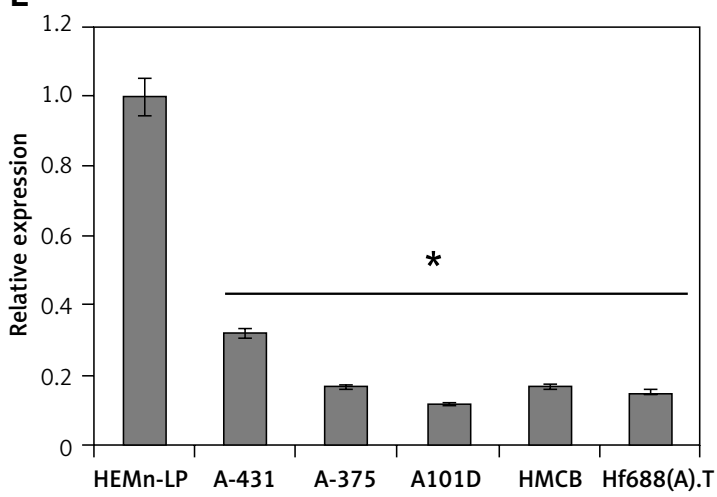

G

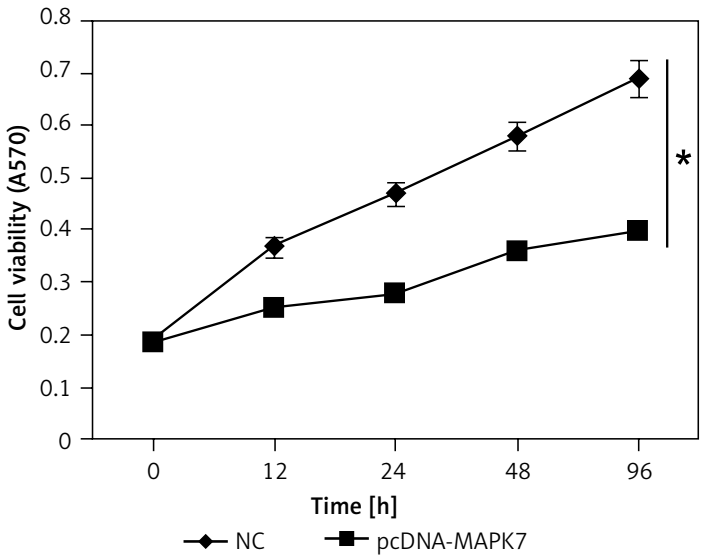

$\rightarrow$ miR-NC $\rightarrow-$ miR-27a $\operatorname{lnh} \rightarrow-m i R-27 a \operatorname{lnh}+$ si-MAPK7

Figure 4. A - Bioinformatic analysis showing MAPK7 as the target of miR-27a. B - Dual luciferase assay. C - Western blot analysis showing expression of MAPK7 in normal and skin cancer cell lines. D - Western blot analysis showing expression of MAPK7 in miR-NC and miR-27a inhibitor transfected A431 cells. E- qRT-PCR analysis showing expression of MAPK7 in normal and skin cancer cell lines. F - qRT-PCR analysis showing expression of MAPK7 in miR-NC and miR-27a inhibitor transfected A431 cells. G - Viability of NC and pcDNA-MAPK7 transfected cells. H - Viability of miR-NC, miR-27a inhibitor and miR-27a inhibitor + MAPK7 transfected A431 cells. The experiments were performed in triplicate and expressed as mean \pm SD $\left({ }^{*} p<0.05\right)$ 
cer are probably caused by a combination of increased exposure to ultraviolet (UV) or sun light, increased outdoor activities, changes in clothing style, increased longevity, ozone depletion, genetics and in some cases, immune suppression [15, 16]. Owing to the discovery of a multitude of roles of miRs in cancer development and progression, their therapeutic implications are being explored for the management of different cancer types [17]. This study showed that transcript levels of miR-27a are aberrantly enhanced in skin cancer tissues and cell lines. A number of previous studies have also revealed the aberrant upregulation of miR-27a in cancer cells. For instance, upregulation of miR-27a has been observed in the case of prostate and colon cancer $[6,18]$. We inhibited the expression of miR-27a to reveal its effects on A431 cell viability and, interestingly, the MTT assay showed time-dependent depletion of A431 cell viability. These findings clearly indicate that miR$27 \mathrm{a}$ acts as an oncomiR in skin cancer. Apoptosis is an important process that is essential for an organism to remove malignant cells [19]. A number of proteins are considered to be biomarkers of apoptosis such as Bax, caspase- 3 and $\mathrm{Bcl}-2$, to name a few [20]. The findings of this study showed that miR-27a suppression promoted apoptosis in the $A 431$ cells as evident from the phase contrast, $A O / E B$ and annexin V/PI staining. The immunohistochemical analysis showed that the $\mathrm{BCl}-2$, p53 and Ki-67 expression decreased upon inhibition of miR-27a. The Bax and $\mathrm{Bcl}-2$ proteins are considered to be biomarker proteins for the induction of apoptosis [12]. Herein, the western blots also showed the alteration in expression of apoptosis-related proteins, as Bax and caspase- 3 increased and $\mathrm{Bcl}-2$ decreased.

A previously carried out research study revealed that miR-27a inhibition led to suppression of the migration and invasion of osteosarcoma cells [10]. This study also revealed that the inhibition of miR27a suppressed the migration and invasion of A431 skin cancer cells. The bioinformatics and dual luciferase assays showed that miR-27a targets MAPK7, which is in consistent with a previous study wherein MAP2K3 was shown to be a target of miR-27a in osteosarcoma [10]. This study revealed that MAPK7 was considerably increased upon suppression of miR-27a. Additionally, MAPK7 overexpression also reduced the A431 cell viability time dependently. Nonetheless, silencing of MAPK7 could avoid the growth inhibitory effects of miR-27a inhibition. This is in agreement with previous studies wherein MAPK7 has been shown to regulate the growth and metastasis of different cancer types [21].

In conclusion, taking the evidence together, miR-27a is considerably upregulated in skin cancer cells. Silencing of miR-27a suppresses the viability of skin cancer cells via promotion of apoptosis. Inhibition of miR-27a also suppresses the migration and invasion of skin cancer cells by targeting MAPK7. miR-27a has therapeutic implications in skin cancer.

\section{Conflict of interest}

The authors declare no conflict of interest.

\section{References}

1. de Gruijl FR. Skin cancer and solar UV radiation: Eur J Cancer 1999; 35: 2003-9.

2. Donaldson MR, Coldiron BM. No end in sight: the skin cancer epidemic continues. In Seminars in cutaneous medicine and surgery. Frontline Medic Commun 2011; 30: 3-5.

3. Aitken JF, Elwood M, Baade PD. Clinical whole-body skin examination reduces the incidence of thick melanomas. Int J Cancer 2010; 126: 450-8.

4. Carthew RW, Sontheimer EJ. Origins and mechanisms of miRNAs and siRNAs. Cell 2009; 136: 642-55.

5. Slaby O, Svoboda M, Fabian P, et al. Altered expression of miR-21, miR-31, miR-24 and miR-145 is related to clinicopathologic features of colorectal cancer. Oncology 2007; 72: 397-402.

6. Tao J, Wu D, Xu B, et al. microRNA-133 inhibits cell proliferation, migration and invasion in prostate cancer cells by targeting the epidermal growth factor receptor. Oncol Rep 2012; 27: 1967-75.

7. Xu R, Han Y. Long non-coding RNA FOXF1 adjacent non-coding developmental regulatory RNA inhibits growth and chemotherapy resistance in non-small cell lung cancer. Arch Med Sci 2019; 15: 1539-46.

8. Liu T, Tang H, Lang Y, Liu M, Li X. MicroRNA-27a functions as an oncogene in gastric adenocarcinoma by targeting prohibitin. Cancer Letters 2009; 273: 233-42.

9. Ma Y, Yu S, Zhao W, Lu Z, Chen J. miR-27a regulates the growth, colony formation and migration of pancreatic cancer cells by targeting Sprouty2. Cancer Letters 2010; 298: 150-8.

10. Pan W, Wang H, Jianwei R, Ye Z. MicroRNA-27a promotes proliferation, migration and invasion by targeting MAP2K4 in human osteosarcoma cells. Cell Physiol Biochem 2014; 33: 402-12.

11. Sun $\mathrm{Q}$, Cong R, Yan $\mathrm{H}$, et al. Genistein inhibits growth of human uveal melanoma cells and affects microRNA-27a and target gene expression. Oncol Rep 2009; 22: 563-7.

12. Hua F, Li CH, Chen XG, Liu XP. Daidzein exerts anticancer activity towards SKOV3 human ovarian cancer cells by inducing apoptosis and cell cycle arrest, and inhibiting the Raf/MEK/ERK cascade. Int J Mol Med 2018; 41: 3485-92.

13. Dong X, Lv B, Li Y, Cheng Q, Su C, Yin G. MiR-143 regulates the proliferation and migration of osteosarcoma cells through targeting MAPK7. Arch Biochem Biophys 2017; 630: 47-53.

14. Berwick M, Halpern A. Melanoma epidemiology. Curr Opin Oncol 1997; 9: 178-82.

15. Apalla Z, Nashan D, Weller RB, Castellsague X. Skin cancer: epidemiology, disease burden, pathophysiology, diagnosis, and therapeutic approaches. Dermatol Therapy 2017; 7: 5-19.

16. Apalla Z, Lallas A, Sotiriou E, Lazaridou E, loannides D. Epidemiological trends in skin cancer. Dermatol Pract Conceptual 2017; 7: 1-6. 
17. Mertens-Talcott SU, Chintharlapalli S, Li X, Safe S. The oncogenic microRNA-27a targets genes that regulate specificity protein transcription factors and the G2-M checkpoint in MDA-MB-231 breast cancer cells. Cancer Res 2007; 67: 11001-11.

18. Chintharlapalli S, Papineni S, Abdelrahim M, et al. Oncogenic microRNA-27a is a target for anticancer agent methyl 2-cyano-3, 11-dioxo-18beta-olean-1, 12-dien30-oate in colon cancer cells. Int J Cancer 2009; 125: 1965-74.

19. Green DR, Reed JC. Mitochondria and apoptosis. Science 1998; 281: 1309-12.

20. Finucane DM, Bossy-Wetzel E, Waterhouse NJ, Cotter TG, Green DR. Bax-induced caspase activation and apoptosis via cytochrome $\mathrm{c}$ release from mitochondria is inhibitable by BclxL. J Biol Chem 1999; 274: 2225-33.

21. Zhou LL, Dong JL, Huang G, Sun ZL, Wu J. MicroRNA-143 inhibits cell growth by targeting ERK5 and MAP3K7 in breast cancer. Brazil J Med Biol Res 2017; 50: e5891. 